\title{
BMJ Open Protocol for a prospective, longitudinal, cohort study of postconcussive symptoms in children: the Take C.A.Re (Concussion Assessment and Recovery Research) study
}

\author{
Silvia Bressan, ${ }^{1,2}$ Michael Takagi, ${ }^{1,3}$ Vicki Anderson, ${ }^{1,3,4,5}$ Gavin A Davis, ${ }^{1}$ \\ Ed Oakley, ${ }^{1,4,6}$ Kevin Dunne, ${ }^{1,4,7}$ Cathriona Clarke, ${ }^{1}$ Melissa Doyle, ${ }^{1}$ \\ Stephen Hearps, ${ }^{1}$ Vera Ignjatovic, ${ }^{1}$ Marc Seal, ${ }^{1}$ Franz E Babl ${ }^{1,4,6}$
}

To cite: Bressan S, Takagi M, Anderson V, et al. Protocol for a prospective, longitudinal, cohort study of postconcussive symptoms in children: the Take C.A.Re (Concussion Assessment and Recovery Research) study. BMJ Open 2016;6:e009427. doi:10.1136/bmjopen-2015009427

- Prepublication history for this paper is available online. To view these files please visit the journal online (http://dx.doi.org/10.1136/ bmjopen-2015-009427).

SB and MT are joint first authors.

Received 17 July 2015 Revised 25 October 2015 Accepted 26 October 2015

CrossMark

For numbered affiliations see end of article.

Correspondence to Prof Vicki Anderson; Vicki.anderson@rch.org.au

\section{ABSTRACT}

Introduction: A substantial minority of children who sustain a concussion suffer prolonged postconcussive symptoms. These symptoms can persist for more than 1 month postinjury and include physical, cognitive, behavioural and emotional changes. Those affected can develop significant disability, diminishing their quality of life. The precise prevalence of postconcussive symptoms following child concussion is unclear, with heterogeneous and at times conflicting results published regarding factors that predict children at risk for developing long-lasting postconcussive symptoms. The aim of the Take C.A.Re (Concussion Assessment and Recovery Research) study is to provide an in-depth multidimensional description of the postconcussive recovery trajectories from a physical, neurocognitive and psychosocial perspective in the 3 months following concussion, with a focus on the early postconcussive period, and identification of factors associated with prolonged recovery.

Methods and analysis: Take C.A.Re is a prospective, longitudinal study at a tertiary children's hospital, recruiting and assessing patients aged 5$<18$ years who present to the emergency department with a concussion and following them at 1-4 days, 2 weeks, 1 month and 3 months postinjury. Multiple domains are assessed: postconcussive symptoms, balance and coordination, neurocognition, behaviour, quality of life, fatigue, post-traumatic stress symptoms, parental distress and family burden. 'Delayed recovery' is operationalised as the presence of $\geq 3$ symptoms on the Post Concussive Symptoms Inventory rated as worse compared with baseline. Main analyses comprise analysis of variance (recovery trajectories, delayed vs normal recovery groups) and regression analyses of predictors of recovery (preinjury, acute and family factors).

Ethics and dissemination: Ethical approval has been obtained through the Royal Children's Hospital Melbourne Human Research Ethics Committee (33122). We aim to disseminate the findings through

\section{Strengths and limitations of this study}

- This study will recruit and follow-up children with concussion from acute emergency department presentation to 3 months, reducing potential bias related to later recruitment.

- It will simultaneously assess multiple domains (clinical factors, child: quality of life, physical, mental health, neurocognition, parent: mental health) which to date have mostly been explored in isolation. This will enable in-depth analysis of postconcussive symptoms and an examination of the factors that place children at risk of prolonged recovery, and their potential interactions.

- As with previous studies, the representative nature of our sample is limited due to the likelihood that not all children present for medical evaluation to the emergency department following a concussion.

international conferences, international peer-reviewed journals and social media.

Trial registration number: ACTRN12615000316505.

\section{INTRODUCTION}

Concussion, a subset of the spectrum of mild traumatic brain injury (TBI), refers to a complex pathophysiological process affecting the brain, induced by biomechanical forces. ${ }^{1}$ Concussions are common among children and adolescents and represent a significant public health issue. Indeed, concussive injuries comprise the majority of presentations for head injuries in hospital emergency departments (ED) in developed countries. ${ }^{2-4}$ For example, in the USA, recent epidemiological data show approximately 1 of every 220 
paediatric patients seen in the ED is diagnosed with a concussion, representing $144000 \mathrm{ED}$ visits annually. ${ }^{5}$ Similarly, in Melbourne, Australia, an audit at The Royal Children's Hospital (RCH) ED identified 1115 children that presented with a head injury over a 12-month period; $90 \%$ of these cases were classified as mild. ${ }^{6}$

Hospitalisation rates and costs related to concussion, particularly sports related, are growing ${ }^{78}$ in part due to increased media focus and community awareness, resulting in more concussed young people seeking medical care. ${ }^{9}$ Previously, concussions were viewed as minor mishaps to be shaken off and presumed not to interfere with daily activities. However, recent research has highlighted significant short and long-term consequences of concussion in children, such as the development of persistent postconcussive symptoms.

Postconcussive symptoms include a constellation of long-term physical, cognitive, emotional and behavioural symptoms, ${ }^{10}{ }^{11}$ for example, headaches, dizziness, visual disturbance, memory/concentration deficits, mental slowness, confusion, fatigability, irritability, light/noise sensitivity and sleep disturbances. ${ }^{12}$ Paediatric studies, mostly comprising participants over 10 years of age have reported an incidence of prolonged postconcussive symptoms varying between $6 \%$ and $59 \%$ in children following a concussion. ${ }^{13-15}$ Secondary mental health problems (eg, anxiety and depression) are also reported. ${ }^{16} 17$ In adults, symptoms generally resolve in $7-10$ days in up to $90 \%$ of cases, ${ }^{18-20}$ but evidence on time to recovery in children is limited.

Studies examining recovery from child concussion report that up to one-third of children remain symptomatic at 1 month, $10 \%$ of children remain symptomatic at 3 months, and less than $5 \%$ are symptomatic at 1 year. ${ }^{11}{ }^{14}$ Children who remain symptomatic long-term can develop significant disability, which may interfere with their school and sporting achievements and social activities. ${ }^{21}$ These difficulties can contribute to the development of more persistent cognitive symptoms, including reduced concentration and memory problems, with impact on the quality of life for the patient and the entire family. Importantly, however, the majority of paediatric concussion studies do not assess participants acutely, with initial evaluations generally performed at 1-3 months postinjury, and often in the context of selfreferral. This approach is problematic as it may result in selection bias, with 'early recoverers' less likely to be captured in study samples, and thus rates of delayed recovery overly inflated. Indeed, in the few studies that have investigated acute recovery in children, symptoms typically resolve within 2 weeks, ${ }^{14}$ in keeping with the adult literature, which describes a substantial portion of individuals to be recovered within 1 month.

Numerous studies have investigated risk factors or predictors for developing prolonged postconcussive symptoms in older children and youth, however, unidimensional approaches (eg, examining only previous concussion or acute clinical variables) and heterogeneous methods have yielded inconsistent results. ${ }^{13} 15 \quad 22-26$ Comprehensive evidence across acute and preinjury time points is necessary to assist clinicians in better advising patients and their families about the expected recovery course postconcussion. In addition, evidence canvassing multiple domains identified as associated with recovery is needed in order to optimise postdischarge multidisciplinary follow-up of children who sustain a concussion.

\section{Objectives}

The main goal of this study is to provide a multidimensional analysis of recovery in children in the first 3 months following the concussion, with a particular focus on the early recovery stage. Specific objectives are to: (A) document recovery trajectories with respect to postconcussive symptoms, physical and cognitive functioning, and mental health (eg, post-traumatic stress symptoms); (B) identify pre-injury, injury-related and early postinjury factors associated with prolonged recovery; (C) examine the relationship between patient clinical symptoms and preinjury function and patient and parent quality of life; and (D) investigate the relationship between family burden and parent mental health on the patient's symptoms.

\section{METHODS AND ANALYSIS}

\section{Study design}

Single-centre prospective longitudinal cohort study.

\section{Study setting}

The Take C.A.Re (Concussion Assessment and Recovery Research) study is conducted in Melbourne, Australia at the Murdoch Childrens Reasearch Institute (MCRI) and the $\mathrm{RCH} . \mathrm{RCH}$ is the primary specialist paediatric hospital for south-eastern Australia and approximately 82000 patients come through the ED per year. MCRI is the research arm of the RCH and the largest child health research institute in Australia. Participant recruitment and the initial assessment will be conducted within the ED of RCH. Follow-up appointments are conducted within a dedicated concussion clinic at MCRI. The concussion clinic is a collaborative initiative between $\mathrm{RCH}$ clinicians (emergency and rehabilitation physicians, neurosurgeons and neuropsychologists) and MCRI clinical researchers (Emergency, Child Neuropsychology, Developmental Neuroimaging and Haematology research groups), with input from other specialties as necessary.

\section{Definitions}

Concussion is defined according to the Zurich consensus statement on Concussion in sport, ${ }^{1}$ which includes the symptoms and signs of a head injury (result of direct or indirect force to the head). To be diagnosed with concussion patients have to present one of more of the following: 
- Somatic symptoms (eg, headache and vomiting).

- Cognitive symptoms (eg, feeling like in fog and slowed reaction times).

- Emotional symptoms (eg, lability and irritability).

- Physical signs (eg, loss of consciousness and amnesia).

- Behavioural changes (eg, irritability).

- Sleep disturbance (eg, insomnia and fatigue, drowsiness).

\section{Study population}

Inclusion criteria

Patients $\geq 5$ and $<18$ years of age who present to the ED with a concussion sustained in the previous $48 \mathrm{~h}$ will be screened for eligibility.

\section{Exclusion criteria}

Patients will be excluded from the study if they present with any of the following: Glasgow Coma Scale (GCS) $<13$; need of neurosurgical operative intervention or general anaesthesia for injury management; presence of structural/haemorrhagic intracranial injury on CT scan of the head; clinical evidence of CSF leak; intellectual disability that impairs completion of the testing measures; non-accidental injury; alcohol or drug intoxication on presentation to $\mathrm{ED}$; insufficient understanding of English as per assessment by the treating physician and research assistant in the ED; multiple trauma; presence of fever at time of initial assessment $\left(\mathrm{T}>37.5^{\circ} \mathrm{C}\right)$; previously enrolled in the study; no clear history of trauma as primary event (eg, trauma following seizure, syncope or migraine).

\section{Patient selection and recruitment}

Patients who are present within the RCH ED between 9:00 and 22:00 7 days per week are screened for eligibility. These hours were chosen based on local data showing most concussion cases present outside of normal office hours (ie, 9:00-17:00). Eligible patients are identified by trained research assistants (RA) through real-time surveillance of the ED's electronic visits database. The RA verifies with the treating clinician whether the patient meets inclusion criteria. If so, the RA discusses the study with the patient and his/her families and invites them to participate. Eligible parents and their children $>12$ years are asked for written informed consent, while younger children will provide assent. RAs keep a screening log of potentially eligible patients presenting within and outside their recruitment shifts. Reasons for exclusion and for possible refusal are recorded.

\section{Initial assessment in the ED}

Consenting participants complete the Sports Concussion Assessment Tool 3rd edition (SCAT3), ${ }^{1}$ under the guidance of a trained RA. The SCAT3 is a multimodal test battery to be used for acute assessment of sport concussions, currently recommended by the Zurich consensus statement on Concussion in sport. The assessment includes a symptom checklist, a cognitive assessment (Standardised Assessment of Concussion, SAC), a neck examination, a balance assessment (Balance Error Scoring System, BESS), and a coordination examination. There are two versions of the SCAT3; one for those aged 13 years and over (SCAT3) and another for children aged 5-12 years (Child-SCAT3). ${ }^{1}$

The SAC and its child version (SAC-C) is a rapid cognitive assessment tool. It includes orientation, immediate memory, concentration and delayed recall tests, ${ }^{27}{ }^{28}$ and is valid for detecting immediate effects of a concussion both in adults ${ }^{29} 30$ and children (Davis GA, et al, 2013 unpublished data).

For the SCAT3, the orientation scale consists of five questions (four questions for the Child SCAT) with possible scores of 1 or 0 , and higher score indicating better performance. For immediate memory, the RA reads aloud a five-word list over three-trials. At the end of each trial, the participant is asked to recall as many words as they remember from the list. Each correct response is awarded a point, with the highest possible score being 15. Following a $10 \mathrm{~min}$ delay, participants are asked to recall as many words as possible from the five-word list and a point is awarded for each correctly recalled word (maximum of five points). For concentration, participants are presented with a series of digits and asked to verbally repeat them in the reverse order in which they were presented. After every presentation, the number of digits increases by 1 and participants are allowed two attempts at each digit span. Correct responses are awarded a score of one point, with a maximum score of four points for digit span. Participants are also asked to recite the months of the year in reverse order (days of the week for the ChildSCAT3) and are awarded a single point for completion. The concentration component has a maximum score of five points (digit span plus months of the year/days of the week backwards).

The BESS assesses postural stability. It includes single, double, and tandem stance assessments, each held for $20 \mathrm{~s}$, with the patient hands on hips and eyes closed. ${ }^{31} 32$ The BESS score represents total errors made by the patient with a maximum of 10 errors for any single condition (total maximum score possible is 20 for children $\leq 12$ years and 30 for older children). Coordination is assessed by the finger-to-nose task that measures the numbers of correct repetitions of the task over $4 \mathrm{~s}$, and scored as 1 (passed) if five correct repetitions are performed in less than $4 \mathrm{~s}{ }^{1}$

Additional clinical information is obtained from the treating clinician who is asked to complete a dedicated clinical report form detailing injury mechanism, clinical findings, previous concussions and ED management.

\section{Follow-up assessments}

Following initial assessment (T0) in the ED, all patients are asked to attend the concussion clinic for follow-up. These appointments include research and clinical 
assessments performed at 1-4 days following ED presentation (T1) and at 2 weeks postinjury (T2). Patients who remain symptomatic at 2 weeks are asked to return to the clinic at 1 month (T3) postinjury, while asymptomatic patients at $\mathrm{T} 2$ are asked to complete either paper or web-based questionnaires at T3, depending on family preference. All patients, independently of their symptoms at T2, are asked to return to the concussion clinic for a final follow-up assessment at 3 months (T4) postinjury.

Research protocols are completed by the patients and their families under the guidance of trained RAs, while clinical assessments are performed by medical and allied health staff from the Rehabilitation and Psychology Services with input from other specialties when appropriate.

A flow-chart describing the study procedures is reported in figure 1 .

\section{Study outcomes}

\section{Primary outcome}

The primary study outcome is the proportion of children aged $5-<18$ years who have persistent postconcussive symptoms at T2, 2 weeks postinjury, defined as the presence of three or more symptoms on the Post Concussive Symptoms Inventory (PCSI) ${ }^{33}$ rated as worse compared with baseline.

\section{Secondary outcomes}

These include (1) postconcussive symptoms at 1 month postinjury; (2) 3 months performances in the following domains: neurocognition, physical recovery, psychosocial/mental health status, quality of life, post-traumatic stress symptoms, family burden; (3) preinjury/ injury-related and early postinjury factors associated with rapid or prolonged recovery will be explored; and (4) timing of return to school/return to sport and association with delayed recovery.

\section{Data collection and research measures}

Data collection will include the National Institutes of Health (NIH) common data elements selection of measures chosen by expert opinion by the Pediatric TBI (Traumatic Brain Injury) Demographics and Clinical Assessment Working Group. ${ }^{34}$ This standardisation of data collection will permit comparison of results and high-quality meta-analysis in the future.

A detailed summary of data collection and timeline is described in table 1 .

\section{Clinical and demographics}

Child and parent age, gender, ethnic background, and education, and parent employment status are recorded. Clinical information on preinjury status to establish patient baseline status includes: hand preference, details of participation in sport; medical history with particular attention to prior concussions and recurrent symptoms, pre-existing sleep disturbances, mental-health status of patients and parents, and completion of the retrospective preinjury PCSI report.

Injury-related clinical data are provided by the treating clinicians in ED in a dedicated clinical report form and include information on injury mechanism (eg, motor vehicle accident and previous concussions), presenting symptoms (eg, loss of consciousness, witnessed disorientation and vomiting), physical examination features (eg, GCS, possible neurologic signs and physical signs of trauma) and ED management. Whether or not the injury was sustained while participating in sport will also be recorded.

\section{Post concussive symptoms evaluation}

The PCSI ${ }^{33}$ includes a set of symptoms scales to be completed by both parents (a self-report 20-item, seven-point Likert scale) and children (5 items, three-point Likert scale for children aged 5-7 years; 17 items, three-point Likert scale for children aged 8-12 years, and a selfreport 20-item, seven-point Likert scale for children aged 13-18 years). The PCSI measures physical, cognitive, emotional and sleep-related symptoms, with lower scores indicating fewer symptoms. The PCSI has strong psychometric characteristics including reliability and validity. ${ }^{35}$

At T1 patients and parents retrospectively complete the preinjury PCSI to collect information on symptoms that were present prior to the injury, as well as to establish the patient baseline, in order to assess the study primary outcome of 'delayed recovery' (defined as a change from baseline of three or more symptoms at 2 weeks postinjury).

\section{Physical domain evaluation}

Physical assessment will include the BESS and the coordination test included in the SCAT3 and Child-SCAT3. ${ }^{1}$

\section{Neurocognitive evaluation}

The, SAC $^{27}$ from the SCAT3, as well as a computerised neurocognitive battery, CogSport ${ }^{36}$ will be administered.

CogSport comprises a series of computerised neurocognitive tests, derived from theoretical and developmentally sensitive principles that have been widely validated in adults and is also appropriate for use in children 5 years and older. ${ }^{36}$ Reliability and metric properties in adults have been documented ${ }^{37} 38$ and adult and child normative data are published. ${ }^{39} 40$

CogSport was developed specifically for application in sport, and concussion in particular. It includes a series of tasks based on a 'playing-card' paradigm presented on a laptop computer fitted with headphones and participants are required to respond by pressing predetermined keys representing 'Yes' or 'No'. Written instructions are presented on the screen to indicate the rule for each task. Once the participant successfully completes a practice trial, the test begins. For each task, 30 correct responses are required before proceeding to the next task. Reaction time and response accuracy are recorded for each trial. Brief descriptions of each task are provided below. 
Figure 1 Flow-chart of study assessments. ED, emergency department; SCAT3, Sports Concussion Assessment Tool 3rd edition.

\section{Excluded patients and drop outs}

Clinical Pathway

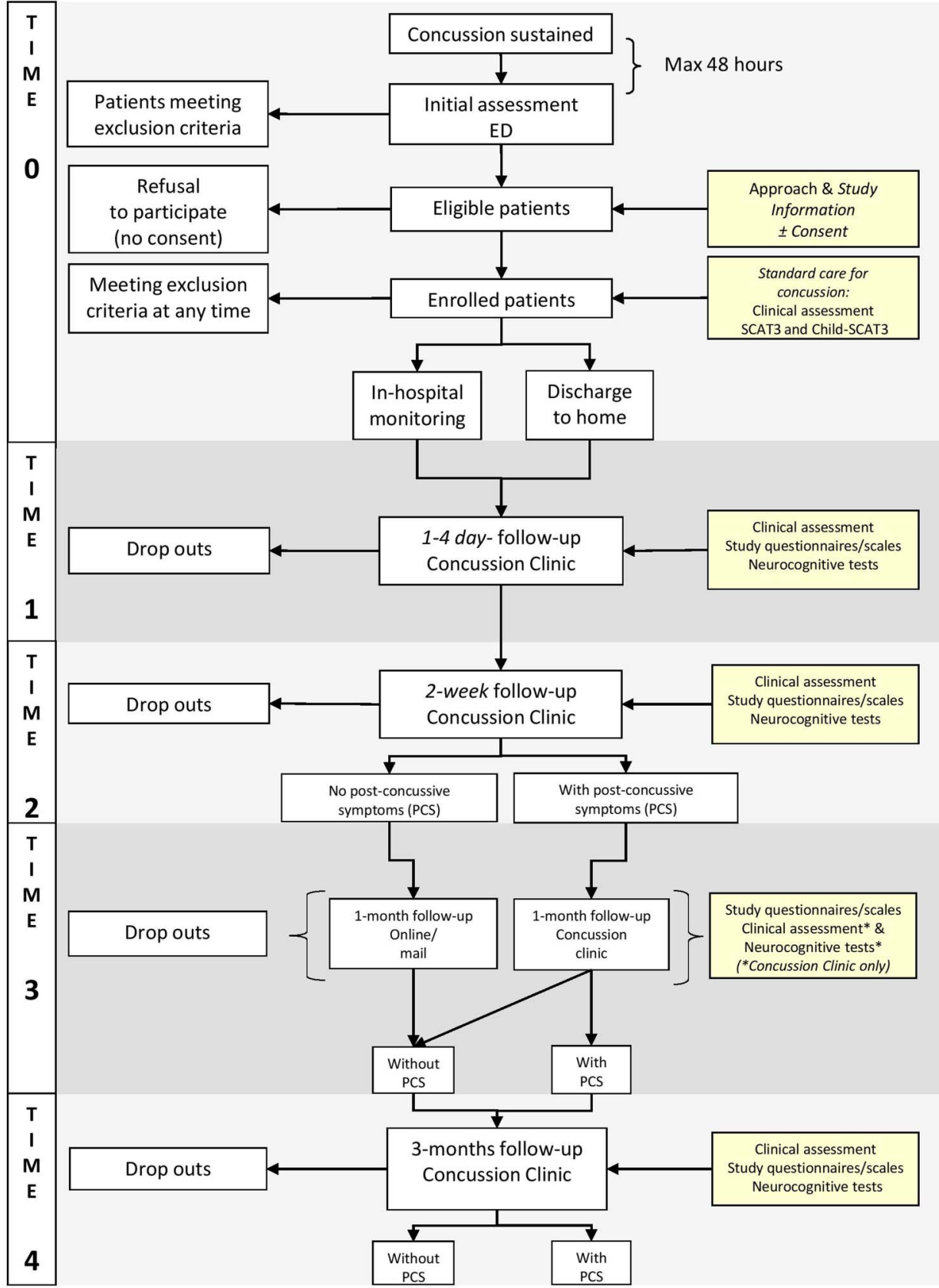

Assessments

Performed
Detection: simple reaction time. A card is presented face down at the centre of the screen. The participant is instructed to 'Press YES' as soon as a card turns face up. After a randomised delay, the next card flips face up, and the participant must press the 'Yes' key as quickly as possible.

Identification: simple decision-making. A card is presented face down at centre screen, and the participant is asked: 'Is the face-up card red?' and responds by pressing 'Yes'. After a randomised delay, the next card flips face up, and the participant must respond 'Yes' or 'No'.
One Back: working memory. A card is presented face up at centre screen. The participant is asked 'Does the face up card exactly match the one before?' If the two cards are identical, the participant presses 'Yes' key, if not, 'No'.

One Card Learning: learning/memory. A card is presented face down at centre screen. The participant is asked 'Have you seen this card before?' After a randomised delay, the card flips face up. Participants must decide whether they have seen that card before and respond 'Yes' or 'No'. 
Table 1 Summary of data collection and timeline

\begin{tabular}{|c|c|c|c|c|c|}
\hline Timeline & $\begin{array}{l}\text { TO } \\
\text { ED presentation }\end{array}$ & $\begin{array}{l}\text { T1 } \\
1-4 \text { days }\end{array}$ & $\begin{array}{l}\text { T2 } \\
2 \text { weeks }\end{array}$ & $\begin{array}{l}\text { T3 } \\
1 \text { month }\end{array}$ & $\begin{array}{l}\text { T4 } \\
3 \text { months }\end{array}$ \\
\hline Demographics & $\mathrm{X}$ & & & & \\
\hline \multicolumn{6}{|l|}{ Preinjury data } \\
\hline Dedicated CRF & & $X$ & & & \\
\hline PCSI (pre-injury-baseline) & & $X$ & & & \\
\hline \multicolumn{6}{|l|}{ Injury-related data } \\
\hline Dedicated CRF & $X$ & & & & \\
\hline \multicolumn{6}{|l|}{ Clinical assessment } \\
\hline SCAT3/Child-SCAT3 & $\mathrm{X}$ & $X$ & $x$ & $(X)$ & $x$ \\
\hline PCSI & & $X$ & $x$ & $x^{\prime}$ & $\mathrm{X}$ \\
\hline \multicolumn{6}{|l|}{ Neurocognitive tests } \\
\hline CogSport & & $\mathrm{X}$ & $x$ & $(\mathrm{X})$ & $\mathrm{X}$ \\
\hline \multicolumn{6}{|l|}{ Depression-anxiety } \\
\hline Child (CBCL) & & & $x$ & & $\mathrm{X}$ \\
\hline Parent (K10) & & & $\mathrm{X}$ & & $\mathrm{x}$ \\
\hline \multicolumn{6}{|l|}{ Quality of life } \\
\hline PedsQL & & $X$ & & $\mathrm{X}$ & $x$ \\
\hline Fatigue scale & & $X$ & & $x$ & $x$ \\
\hline \multicolumn{6}{|l|}{ PTS symptoms } \\
\hline CPSS & & & $\mathrm{X}$ & $\mathrm{X}$ & $x$ \\
\hline \multicolumn{6}{|l|}{ Family burden } \\
\hline Dedicated CRF & & & & $\mathrm{X}$ & $x$ \\
\hline
\end{tabular}

CogSport is appropriate and reliable for administration to children as young as 5 years of age. ${ }^{40} 41$ Paediatric normative data have recently been established by a group of researchers including one of the co-investigators. $^{42}$

\section{Psychosocial domain evaluation}

Child Behavior will be evaluated using the Child Behavior Checklist (CBCL), ${ }^{43}$ a parent rating scale of children's behaviour with the following summary scales: Internalising Behavior, Externalising Behavior, and Total Behavior Score $(\mathrm{M}=50, \quad \mathrm{SD}=10)$, and subscales: Emotionally Reactive, Anxious/depressed, Somatic Complaints, Withdrawn, Sleep Problems, Attention Problems, Aggressive Behavior, and Other Problems. The CBCL consists of 113 items and is scored using a three-point Likert scale. Raw scores are converted to $\mathrm{T}$ scores, a standard score that allows comparison between the subscales. A clinically significant score for any subscale would be a $\mathrm{T}$ Score $>60$. The CBCL is a widely used and validated measure and it is currently recommended by the by the NIH Pediatric Common Data Element TBI Outcomes Workgroup. ${ }^{44}$

Child post-traumatic stress. Children's stress reactions are measured with the Child Post Traumatic Stress Symptom Scale (CPSS), ${ }^{45}$ for children aged between 8 and 18 years. The CPSS is a 24-item measure that can be used in structured interview format. The CPSS yields a continuous severity score, an impairment score, and a probable post-traumatic stress disorder status. The scale has shown excellent internal consistency, test-retest reliability and convergent validity. ${ }^{45} 46$

Child quality of life. The Pediatric Quality of Life Inventory (PedsQL) is a 23-item measure that is used to assess quality of life in children. ${ }^{47}$ It includes physical, emotional, social and school functioning domains. Respondents indicate how much each item has been a problem in the past month; responses for 8-18-year-old children and for parents are rated in a five-point Likert scale, while younger children rate their responses on a three-point scale. A total score and two summary scores are derived for physical health and psychosocial health (1100 ), with higher scores indicating a higher health-related quality of life. This tool is recommended by the NIH Paediatric Common Data Element TBI Outcomes Workgroup. $^{44}$ The PedsQL multidimensional fatigue scale $^{48}$ is also administered with the standard PedsQL inventory. The PedsQL fatigue measure is an 18-item scale that examines general fatigue, cognitive fatigue and sleep-related fatigue. It is scored in an identical manner to the PedsQL and a total score and summary scores are provided for general, cognitive and sleep-related fatigue.

Parent mental health will be assessed with the Kessler Psychological Distress Scale (K10), ${ }^{49}$ a 10 -item global measure of parent distress in the previous month. It is scored using a five-point Likert scale, with a higher score indicating more frequent symptoms. A score $>19$ indicates significant psychological distress. 
Family Burden. Data collection to assess family burden includes number of days of school missed because of postconcussive symptoms, number of sport training sessions missed-for children involved in organised sport activities, number of missed days of work for parents or need for an additional carer to look after children who could not attend school because of symptoms.

\section{Data analysis}

Participant demographics and injury characteristics will be analysed using descriptive statistics. Recovery will be dichotomised into 'normal' and 'delayed' recovery. Based on PCSI scores, delayed recovery will be operationalised as the persistence of postconcussive symptoms at T2, defined as the presence of three or more symptoms on the PCSI compared to baseline (ie, T2-T1 PCSI Scores $>3$ ).

We will explore associations between the primary outcome, postconcussive symptoms and secondary outcomes using repeated ordinary least squares regression or logistic regression models, as appropriate. In follow-up, multiple comparison-adjusted tests will be carried out to further examine within-time changes. Potential confounds (such as time since injury) will be investigated and included in all models as covariates when found to significantly predict outcome. We will also examine associations between the primary outcome of postconcussive symptoms at $\mathrm{T} 2$ and preinjury and acute clinical characteristics and family/parent variables. ${ }^{50}$ All characteristics will be analysed in a bivariate logistic regression models. Significant predictors from bivariate analysis (at $\mathrm{p}<0.1$ ) will then be included in a multivariate logistic regression model. Owing to overlap of assessments, multicollinearity of predictors will be tested and addressed in multivariable models.

To consider clinically useful predictors of recovery, we will use our previously defined definition of delayed recovery to dichotomise participants into 'normal' and 'delayed' recovery groups to conduct group comparisons with respect to patient clinical symptoms, preinjury function and patient, parent quality of life, family burden and parent mental health.

Given the expected primary outcome rate of $30 \%$ delayed recovery at 2 weeks postconcussion (as indicated by our pilot data), we would require 60 participants to be categorised as demonstrating delayed recovery at 2 weeks postconcussion (T2) to test a model including six independent variables. This leads to an overall sample size of 200. With this sample size of 200, tests modelling trajectory of neuropsychological outcomes over two or four time points (depending on the outcome) will allow for the detection of small effect sizes $($ Cohen's $\mathrm{f}<0.1)$. All tests employ an $\alpha$ level of 0.05 , and $80 \%$ power.

\section{Methodological considerations}

Identifying clinically significant concussions

The pilot study for the present protocol highlighted important methodological considerations, one of them being the difficulties associated with determining a clinically significant concussion using a standardised instrument (ie, the PCSI). The PCSI is a valid and reliable measure for identifying the range and severity of concussion symptoms. ${ }^{35}$ The definition we are using for the present study is the presence of three or more symptoms on the PCSI rated as worse compared with baseline, however, there is not an established definition in the literature to reflect the presence of symptoms that are clinically significant for the patient. In addition, baseline/ preinjury data are collected retrospectively and thus subject to bias. Given our data collection, we will be able to explore the clinical utility of this definition of recovery, and others (eg, including the presence of a single elevated postconcussive symptom, such as severe headache or excessive fatigue) by correlating each scenario with quality of life outcomes. With the present study we will also explore the patient and family burden related to prolonged postconcussive symptoms in order to refine the procedures for identifying clinically significant postconcussive symptoms in children.

\section{Bias in recruitment}

The nature of the present study requires a significant commitment from participants. Parents and children are asked to attend the hospital on at least three separate occasions for approximately $1 \mathrm{~h}$ each visit. Many eligible participants who are approached in the ED are interested in participating, but are unable to commit to the study due to other time commitments. It is therefore possible we are recruiting a biased sample (ie, families who can afford to bring their child in). To address this issue, current efforts are directed towards expanding the hours of the study and enhancing the online component, where participants may complete an abbreviated battery of questionnaires at home, either online or via an App, and their responses will be monitored by clinical staff.

\section{ETHICS AND DISSEMINATION}

The study poses little to no risk to participants and their families. Signed informed consent is obtained from all participating families. Participation in the study does not interfere with the typical care patients receive in the ED (eg, CT head). Clinical follow-up is provided by clinicians in the concussion clinic and, if necessary, participants are referred on for additional clinical management (eg, rehabilitation, neurosurgery, clinical psychology).

Results from this study will be disseminated at regional and international conferences and in peer-reviewed journals.

\section{SIGNIFICANCE AND OUTLOOK}

The study will provide in depth insight into the recovery pattern of children who sustain a concussion, beginning at acute presentation for medical care and using a multidimensional perspective. Study results will inform 
clinicians, patients and their families on the expected course of postconcussive symptoms recovery. Detailed understanding of the importance of preinjury, injury-related and early postinjury factors for either rapid or prolonged recovery will assist in targeting patients at high risk of delayed recovery, who are most likely to benefit from strict monitoring and specific interventions and thus will guide optimal clinical care and follow-up.

In order to better understand the peculiar multidimensional recovery pattern typical of concussion, we plan to expand the study to include a comparison group of children with orthopaedic injuries. We will then plot and contrast recovery pathways for children with concussion and orthopaedic injury controls in the different domains (A) postconcussive symptoms (B) cognition (C) physical function, (D) mental health and quality of life.

In addition to the different domains explored by the present study, we plan to expand the study to include serial collection of plasma biomarkers and MRI sequences. The study of plasma biomarkers will include targeted testing of biomarkers previously shown to be important in this setting and will also explore as yet unknown biomarkers using untargeted proteomic techniques. This will allow for discovery of proteins not yet implicated in concussion research. The MRI sequences will include structural and functional components. The addition of these biological dimensions adds novelty to our approach and will expand on our study objectives to include a focus on understanding mechanisms underpinning recovery from paediatric concussion.

\author{
Author affiliations \\ ${ }^{1}$ Murdoch Childrens Research Institute, Melbourne, Victoria, Australia \\ 2Department of Women's and Children's Health, University of Padova, Padova, \\ Italy \\ ${ }^{3}$ School of Psychological Sciences, University of Melbourne, Victoria, \\ Australia \\ ${ }^{4}$ Department of Paediatrics, University of Melbourne, Melbourne, Victoria, \\ Australia \\ ${ }^{5}$ Psychology Service, The Royal Children's Hospital, Melbourne, Victoria, \\ Australia \\ ${ }^{6}$ Emergency Department, Royal Children's Hospital Melbourne, Victoria, \\ Australia \\ ${ }^{7}$ Department of Rehabilitation Medicine, Royal Children's Hospital, Melbourne, \\ Victoria, Australia
}

Acknowledgements The authors would like to thank participating families and emergency department staff for their support of this study. The authors thank the volunteers and research assistants who have supported this study: Dean Ridings, Kate Stephens, Lucy Davenport, Kelleyne Yu, Rohan Navari, Khalid Elsaafien, Chloe Bradford, Monica Nixon, Diana Dionisio, Elly Ganakas, Stephanie Gauci, Bethany Scott, Claire-Marie Heaney, Lena Stoiber, Jennifer Snowball, Mark Zhong, Sharoan Selvakumaran, Bleydy Dimech-Betancourt, Sabrina Orlandea, On Zhi Xiang, Stephanie Malarbi, Evelyn Deutscher, Harriet Wills, Nick Ryan, Georgia Parkin, Roshan Karri, Lucy Ymer, Jonathan Reyes, Sean McWhirter, Kim Luong, Jennifer Nguyen, Katie Truss, Monica Nation, Chloe Lanyon, Sophie Lijdsman, Torun Finnengar, Meg Hickie, Nick Anderson, Brendan Stevenson, Ali Mcllroy, and Vanessa Rausa.

Contributors SB designed the study with input from all the authors. SB and $M T$ drafted the manuscript and contributed equally to the present work. GAD, FEB, EO, KD, CC, MD, SH, VI, MS and VA significantly contributed to the establishment and refinement of study procedures and critically revised the manuscript. All authors approved the final version of the manuscript.

Funding The study is funded by a project grant from Royal Children's Hospital Foundation, Melbourne, Australia (2014-370). The study is part funded by an Australian National Health and Medical Research Council Centre of Research Excellence for Paediatric Emergency Medicine grant (GNT 1058560) and received infrastructure support from the Victorian Government's Infrastructure Support Program, Melbourne, Australia. FEB is part funded by a Royal Children's Hospital Foundation grant, Melbourne, Australia (2014-400). VA is part-funded by NHMRC Senior Practitioner fellowship.

\section{Competing interests None declared.}

Ethics approval The study has been approved by the Human Research Ethics Committee of the Royal Children's Hospital Melbourne (study number 33122).

Provenance and peer review Not commissioned; externally peer reviewed.

Open Access This is an Open Access article distributed in accordance with the Creative Commons Attribution Non Commercial (CC BY-NC 4.0) license, which permits others to distribute, remix, adapt, build upon this work noncommercially, and license their derivative works on different terms, provided the original work is properly cited and the use is non-commercial. See: http:// creativecommons.org/licenses/by-nc/4.0/

\section{REFERENCES}

1. McCrory $\mathrm{P}$, Meeuwisse WH, Aubry M, et al. Consensus statement on concussion in sport: the 4th International Conference on Concussion in Sport held in Zurich, November 2012. Br J Sports Med 2013;47:250-8.

2. Bazarian JJ, McClung J, Shah MN, et al. Mild traumatic brain injury in the United States, 1998-2000. Brain Inj 2005;19:85-91.

3. Peden M, Oyebite K, Ozanne-Smith J. World report on child injury prevention. Geneva: World Health Organization, 2008.

4. Quayle KS, Powell EC, Mahajan P, et al. Epidemiology of blunt head trauma in children in U.S. emergency departments. N Engl J Med 2014;371:1945-7.

5. Meehan WP III, Mannix R. Pediatric concussions in United States emergency departments in the years 2002 to 2006. J Pediatr 2010;157:889-93.

6. Crowe L, Babl F, Anderson V, et al. The epidemiology of paediatric head injuries: data from a referral centre in Victoria, Australia. $J$ Paediatr Child Health 2009;45:346-50.

7. Lyttle MD, Crowe L, Oakley E, et al. Comparing CATCH, CHALICE and PECARN clinical decision rules for paediatric head injuries. Emerg Med J 2012;29:785-94.

8. Finch CF, Clapperton AJ, McCrory P. Increasing incidence of hospitalisation for sport-related concussion in Victoria, Australia. Med J Aust 2013;198:427-30.

9. Haran HP, Bressan S, Oakley E, et al. On-field management and return-to-play in sports-related concussion in children: are children managed appropriately? J Sci Med Sport 2015.

10. Blume $\mathrm{H}$, Hawash $\mathrm{K}$. Subacute concussion-related symptoms and postconcussion syndrome in pediatrics. Curr Opin Pediatr 2012;24:724-30.

11. Barlow KM, Crawford S, Stevenson A, et al. Epidemiology of postconcussion syndrome in pediatric mild traumatic brain injury. Pediatrics 2010;126:e374-81.

12. Ayr LK, Yeates KO, Taylor HG, et al. Dimensions of postconcussive symptoms in children with mild traumatic brain injuries. J Int Neuropsychol Soc 2009;15:19-30.

13. Zemek RL, Farion KJ, Sampson M, et al. Prognosticators of persistent symptoms following pediatric concussion: a systematic review. JAMA Pediatr 2013:167:259-65.

14. Eisenberg MA, Meehan WP III, Mannix R. Duration and course of post-concussive symptoms. Pediatrics 2014;133:999-1006.

15. Wiebe DJ, Collins MW, Nance ML. Identification and validation of prognostic criteria for persistence of mild traumatic brain injury-related impairment in the pediatric patient. Pediatr Emerg Care 2012;28:498-502.

16. Chrisman SP, Richardson LP. Prevalence of diagnosed depression in adolescents with history of concussion. J Adolesc Health 2014;54:582-6. 
17. Hajek CA, Yeates KO, Taylor HG, et al. Agreement between parents and children on ratings of post-concussive symptoms following mild traumatic brain injury. Child Neuropsychol 2011;17:17-33.

18. Broglio SP, Puetz TW. The effect of sport concussion on neurocognitive function, self-report symptoms and postural control: a meta-analysis. Sports Med 2008;38:53-67.

19. Echemendia RJ, Iverson GL, McCrea $M$, et al. Advances in neuropsychological assessment of sport-related concussion. $\mathrm{Br} \mathrm{J}$ Sports Med 2013;47:294-8.

20. Williams RM, Puetz TW, Giza CC, et al. Concussion recovery time among high school and collegiate athletes: a systematic review and meta-analysis. Sports Med 2015;45:893-903.

21. Ransom DM, Vaughan CG, Pratson L, et al. Academic effects of concussion in children and adolescents. Pediatrics 2015;135:1043-50.

22. Babcock L, Byczkowski T, Wade SL, et al. Predicting postconcussion syndrome after mild traumatic brain injury in children and adolescents who present to the emergency department. JAMA Pediatr 2013;167:156-61.

23. Grubenhoff JA, Deakyne SJ, Brou L, et al. Acute concussion symptom severity and delayed symptom resolution. Pediatrics 2014;134:54-62.

24. Yeates KO, Taylor HG, Rusin J, et al. Premorbid child and family functioning as predictors of post-concussive symptoms in children with mild traumatic brain injuries. Int J Dev Neurosci 2012;30:231-7.

25. McNally KA, Bangert $B$, Dietrich $A$, et al. Injury versus noninjury factors as predictors of postconcussive symptoms following mild traumatic brain injury in children. Neuropsychology 2013;27:1-12.

26. Eisenberg MA, Andrea J, Meehan W, et al. Time interval between concussions and symptom duration. Pediatrics 2013;132:8-17.

27. McCrea M, Kelly JP, Randolph C, et al. Standardized assessment of concussion (SAC): on-site mental status evaluation of the athlete. $\checkmark$ Head Trauma Rehabil 1998;13:27-35.

28. Grubenhoff JA, Kirkwood M, Gao D, et al. Evaluation of the standardized assessment of concussion in a pediatric emergency department. Pediatrics. 2010;126:688-95.

29. Barr WB, McCrea M. Sensitivity and specificity of standardized neurocognitive testing immediately following sports concussion. J Int Neuropsychol Soc 2001;7:693-702.

30. Naunheim RS, Matero D, Fucetola R. Assessment of patients with mild concussion in the emergency department. J Head Trauma Rehabil 2008;23:116-22.

31. Finnoff JT, Peterson VJ, Hollman JH, et al. Intrarater and interrater reliability of the Balance Error Scoring System (BESS). PM $R$ 2009;1:50-4.

32. Guskiewicz KM. Assessment of postural stability following sport-related concussion. Curr Sports Med Rep 2003;2:24-30.

33. Gioia GA, Schneider JC, Vaughan CG, et al. Which symptom assessments and approaches are uniquely appropriate for paediatric concussion? Br J Sports Med 2009;43(Suppl 1):i13-22.

34. Adelson PD, Pineda J, Bell MJ, et al. Common data elements for pediatric traumatic brain injury: recommendations from the working group on demographics and clinical assessment. J Neurotrauma 2012;29:639-53.

35. Sady MD, Vaughan CG, Gioia GA. Psychometric characteristics of the postconcussion symptom inventory in children and adolescents. Arch Clin Neuropsychol 2014;29:348-63.

36. Collie A, Maruff P, Makdissi M, et al. CogSport: reliability and correlation with conventional cognitive tests used in postconcussion medical evaluations. Clin J Sport Med 2003;13:28-32.

37. Falleti MG, Maruff P, Collie A, et al. Qualitative similarities in cognitive impairment associated with $24 \mathrm{~h}$ of sustained wakefulness and a blood alcohol concentration of $0.05 \%$. J Sleep Res 2003;12:265-74.

38. Maruff $P$, Falleti MG, Collie A, et al. Fatigue-related impairment in the speed, accuracy and variability of psychomotor performance: comparison with blood alcohol levels. J Sleep Res 2005;14:21-7.

39. Straume-Naesheim TM, Andersen TE, Bahr R. Reproducibility of computer based neuropsychological testing among Norwegian elite football players. Br J Sports Med 2005;39(Suppl 1):i64-9.

40. Betts J, McKay J, Maruff P, et al. The development of sustained attention in children: the effect of age and task load. Child Neuropsychol 2006;12:205-21.

41. Mollica CM, Maruff P, Collie A, et al. Repeated assessment of cognition in children and the measurement of performance change. Child Neuropsychol 2005;11:303-10.

42. Williams J, Crowe LM, Dooley J, et al. Developmental trajectory of information-processing skills in children: computer-based assessment. Appl Neuropsychol Child 2014:1-9.

43. Achenbach TM, Ruffle TM. The Child Behavior Checklist and related forms for assessing behavioral/emotional problems and competencies. Pediatr Rev 2000;21:265-71.

44. McCauley SR, Wilde EA, Anderson VA, et al. Recommendations for the use of common outcome measures in pediatric traumatic brain injury research. J Neurotrauma 2012;29:678-705.

45. Foa EB, Johnson KM, Feeny NC, et al. The child PTSD Symptom Scale: a preliminary examination of its psychometric properties. J Clin Child Psychol 2001;30:376-84.

46. Kassam-Adams N, Garcia-Espana JF, Marsac ML, et al. A pilot randomized controlled trial assessing secondary prevention of traumatic stress integrated into pediatric trauma care. J Trauma Stress 2011;24:252-9.

47. Varni JW, Seid M, Rode CA. The PedsQL: measurement model for the pediatric quality of life inventory. Med Care 1999;37:126-39.

48. Varni JW, Burwinkle TM, Katz ER, et al. The PedsQL in pediatric cancer: reliability and validity of the Pediatric Quality of Life Inventory Generic Core Scales, Multidimensional Fatigue Scale, and Cancer Module. Cancer 2002;94:2090-106.

49. Kessler RC, Andrews G, Colpe LJ, et al. Short screening scales to monitor population prevalences and trends in non-specific psychological distress. Psychol Med 2002;32:959-76.

50. Harrell FE Jr, Lee KL, Mark DB. Multivariable prognostic models: issues in developing models, evaluating assumptions and adequacy, and measuring and reducing errors. Stat Med 1996;15:361-87. 